\title{
WestVirginiaUniversity
} THE RESEARCH REPOSITORY @ WVU

Graduate Theses, Dissertations, and Problem Reports

2002

\section{Degree sequences}

\author{
Rong Luo \\ West Virginia University
}

Follow this and additional works at: https://researchrepository.wvu.edu/etd

\section{Recommended Citation}

Luo, Rong, "Degree sequences" (2002). Graduate Theses, Dissertations, and Problem Reports. 1235. https://researchrepository.wvu.edu/etd/1235

This Thesis is protected by copyright and/or related rights. It has been brought to you by the The Research Repository @ WVU with permission from the rights-holder(s). You are free to use this Thesis in any way that is permitted by the copyright and related rights legislation that applies to your use. For other uses you must obtain permission from the rights-holder(s) directly, unless additional rights are indicated by a Creative Commons license in the record and/ or on the work itself. This Thesis has been accepted for inclusion in WVU Graduate Theses, Dissertations, and Problem Reports collection by an authorized administrator of The Research Repository @ WVU. For more information, please contact researchrepository@mail.wvu.edu. 


\title{
Degree Sequences
}

\author{
by \\ Rong Luo
}

THESIS

Submitted to the College of Engineering and Mineral Resources

Of

West Virginia University

in partial fulfillment of the requirements

for the degree of

Master of Science

in

Computer Science

Elaine M. Eschen, Ph.D., Chair

Mark Shereshevsky, Ph.D.

Cun-Quan Zhang Ph.D.

Morgantown, West Virginia, 2002

Keywords: graphic sequences, potentially $C_{k}$-graphic, realizations Copyright 2002 Rong Luo 


\section{Acknowledgements}

I would like to thank my supervisor, Dr. Elaine M. Eschen, for her guidance, advice, and continual encouragement. It is my pleasure to work under her supervision. Without her, this thesis could not have come out. I would also like to thank other committee members: Dr. Mark Shereshevsky and Dr. C.Q. Zhang, for their help during my studies.

Finally, I thank my wife, Fang, for her love and her never ending support. 


\section{Contents}

1 Introduction $\quad 1$

1.1 Notations and Definitions . . . . . . . . . . . . . . . . 1

1.2 An Algorithmic Characterization . . . . . . . . . . . . . . . . 2

1.3 Potentially $P$-graphic Sequences $\ldots \ldots \ldots \ldots$

2 Potentially $H$-graphic Sequences $\quad 4$

2.1 Introduction . . . . . . . . . . . . . . . . . . . . . . 4

2.2 Lemmas . . . . . . . . . . . . . . . . . . . . . 5

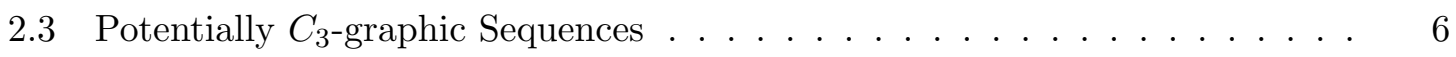

2.4 Potentially $C_{4}$-graphic Sequences $\ldots \ldots \ldots \ldots$

2.5 Potentially $C_{5}$-graphic Sequences $\ldots \ldots \ldots \ldots$. . . . . . . . . . . . . . . . . . . .

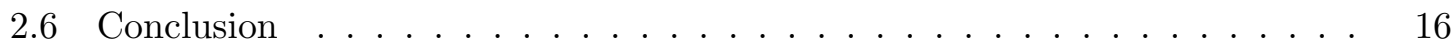




\begin{abstract}
A nonincreasing sequence $\pi=\left(d_{1}, d_{2}, \cdots, d_{n}\right)$ of nonnegative integers is said to be graphic if it is the degree sequence of a simple graph of order $n$ and such graph is referred to as a realization of $\pi$. Let $H$ be a simple graph. A graphic sequence $\pi$ is said to be potentially $H$ graphic if it has a realization $G$ containing $H$ as its subgraph. In this paper, we characterize the potentially $C_{k}$ graphic sequence for $k=3,4,5$. These characterizations imply theorems due to P. Erdös, M. S. Jacobson and J. Lehel [3], R. J. Gould, M. S. Jacobson and J. Lehel[5], and C. H. Lai [16] [17].
\end{abstract}




\section{Chapter 1}

\section{Introduction}

\subsection{Notations and Definitions}

In this thesis, all the graphs are simple. A nonincreasing sequence $\pi=\left(d_{1}, d_{2}, \cdots, d_{n}\right)$ of nonnegative integers is said to be graphic if it is the degree sequence of a simple graph of order $n$ and such graph is referred to as a realization of $\pi$. We use $G(\pi)$ to denote the set of all non-isomorphic realizations of $\pi$.

For $\pi=\left(d_{1}, d_{2}, \cdots, d_{n}\right), \sigma(\pi)=d_{1}+d_{2}+\cdots+d_{n}$ the degree sum of $\pi$. The residual sequence $\pi^{\prime}$ obtained by laying off $d_{k}$ is defined as follows:

$$
\pi^{\prime}= \begin{cases}\left(d_{1}-1, \cdots, d_{k-1}-1, d_{k+1}-1, \cdots, d_{d_{k}+1}-1, d_{d_{k}+2}, \cdots, d_{n}\right), & \text { if } d_{k} \geq k, \\ \left(d_{1}-1, \cdots, d_{d_{k}}-1, d_{d_{k}+1}, \cdots, d_{k-1}, d_{k+1}, \cdots, d_{n}\right), & \text { if } d_{k} \leq k-1 .\end{cases}
$$

It is easy to see that any two isomorphic graphs have the same degree sequence. But the converse is not true. Therefore, the degree sequence is an invariant of graphs under isomorphisms and it is a rather weak invariant. Hence, degree sequences partly reflect some features of graphs, and the extent of this reflection deserves studying.

An interesting and important question on graphic sequences is: "how to characterize the graphic sequences?". This question and its various generalizations were settled by several graph theorists. Sierksma-Hoogeveen[31] lists seven characterizations. In Section 2, we will present an algorithmic characterization due to Senion[30], Havel[7], Hakim[6] and Kleitman and Wang[8]. 


\subsection{An Algorithmic Characterization}

In this section, we present an algorithmic characterization due to Senion[30], Havel[7], Hakim[6] and Kleitman and Wang[8]. The proof is due to Kleitman and Wang[8].

Theorem 1.2.1 The sequence $\pi$ is graphic if and only if the residual sequence $\pi^{\prime}$ is also graphic. Moreover, a realization $G$ of $\pi$ can be obtained from a realization $G^{\prime}$ of $\pi^{\prime}$ by adding a new vertex $v_{k}$ to $G^{\prime}$ and joining it to the vertices whose degrees are reduced by one in going from $\pi$ to $\pi^{\prime}$.

Proof. Suppose that $\pi$ is graphic. It is sufficient to prove that there exists a realization $G$ of $\pi$ with the vertex set $V(G)=\left\{v_{1}, \cdots, v_{n}\right\}$ such that $v_{k}$ is adjacent to the first $d_{k}$ vertices other than itself and $d_{i}=d\left(v_{i}\right)$. If not, we may choose a graph $G \in G(\pi)$ such that the number of vertices adjacent to $v_{k}$ among the first $d_{k}$ vertices other than $v_{k}$ is maximum. Let $v_{i}$ be a vertex among these first $d_{k}$ vertices that is not adjacent to $v_{k}$, and let $v_{j}$ be a vertex not among these first $d_{k}$ vertices that is adjacent to $v_{k}$. We may assume that $d_{i}>d_{j}$, otherwise, the order of $i$ and $j$ can be interchanged. Hence there is a vertex $v_{t}, t \neq i, j$, such that $v_{i} v_{t} \in E(G)$ and $v_{t} v_{j} \notin E(G)$. Clearly, $G^{*}=G-v_{j} v_{k}-v_{i} v_{t}+v_{i} v_{k}+v_{j} v_{t} \in G(\pi)$, and the number of vertices adjacent to $v_{k}$ among the first $d_{k}$ vertices other than $v_{k}$ in $G^{*}$ is larger than that of $G$. This contradicts the choice of $G$.

Conversely, suppose that $\pi^{\prime}$ is graphic. Let $G^{\prime}$ be a realization of $\pi^{\prime}$. Adding a new vertex $v_{k}$ to $G^{\prime}$ and joining it to the vertices whose degrees are decreased by one, the resulting graph $G$ is a realization of $\pi$.

Theorem 1.2.1 is the basis of the following algorithm for determining whether a nonnegative integer sequence is graphic, and if it is, constructing a realization.

Algorithm: Determine whether a nonnegative integer sequence $\pi=\left(d_{1}, d_{2}, \cdots, d_{n}\right\}$ with $d_{1} \geq d_{2} \geq \cdots \geq d_{n}$ is graphic.

(a) If $d_{1} \geq n$, then $\pi$ is not graphic. Otherwise, remove any zero terms from the sequence and then go to (b).

(b) If the resulting sequence is of length zero, then the sequence is graphic. If the resulting sequence is of length 1 , then the sequence is not graphic. Otherwise, go to (c).

(c) Lay off the first number from the sequence.

(d) Rearrange the order of integers in the sequence so that it is nonincreasing and set $n=n-1$ and go to (a). 


\subsection{Potentially $P$-graphic Sequences}

Let $P$ be a graph-theoretic property. Following Nash-Williams[27], we say that a graphic sequence $\pi$ is potentially $P$-graphic if there exists a simple graph $G \in G(\pi)$ such that $G$ has the property $P$.

The first question on potentially $P$-graphic sequences was solved by Edmonds[1], where $P$ is $k$-edge connectedness.

Theorem 1.3.1 (Edmonds[1]) A graphic sequence $\pi=\left(d_{1}, d_{2}, \cdots, d_{n}\right)$ is potentially $k$ edge connected if and only if

(1) $d_{i} \geq k$ for all $i$, and

(2) $\sum_{i=1}^{n} d_{i} \geq 2(n-1)$ if $k=1$.

A graphic sequence is potentially $k$-connected if it has a $k$-connected realization. S. B. Rao and A. R. Rao[29] first investigated the problem concerning the charaterization of the potentially 3-connected graphic sequences. Wang and Kleitman[32] solved this problem for general $k$ by using the laying off operation.

A graphic sequence $\pi$ is said to be unigraphic if every two realizations of $\pi$ are isomorphic. Many authors (for example, Hakimi[6], Johnson[9][10][11], Koren[13][14][15] and S. Y. R. $\mathrm{Li}[24])$ studied the unigraphic sequences. Various charaterizations of unigraphic sequences have been obtained in Kleitman and S. Y. R. Li[12], S. Y. R. Li[24] and Koren[14]. The following is one of the most important and interesting results on the unigraphic sequences.

Theorem 1.3.2 (Koren[15]) A graphic sequence $\pi=\left(d-1, d_{2}, \cdots, d_{n}\right)$ with $d_{2}=d_{n-1}$ is unigraphic if and only if one of the following conditions holds:

(1) $d_{1}=d_{n}=k$, where $k=1, n-1, n-2$;

(2) $d_{1}=d_{n}=2$ and $n=5$;

(3) $d_{1}>d_{2}=d_{n-1}=1$;

(4) $d_{1}>d_{2}=d_{n}=2$ where $d_{1}=n-1$, or, $n-2$;

(5) $n-2=d_{1}=d_{n-1}>d_{n}$;

(6) $n-3=d_{1}=d_{n-1}>d_{n}=1$;

(7) $n-1=d_{1}>d_{2}=d_{n}=3, n=6$;

(8) $n-1=d_{1}>d_{2}=d_{n}=n-2$.

Some other potentially $P$-graphic sequences have also been studied such as potentially $\left(k_{1}, \cdots, k_{n}\right)$-factorable graphic sequences, potentially planar-graphic sequences, potentially Hamiltonian-graphic sequences and so on. 


\section{Chapter 2}

\section{Potentially $H$-graphic Sequences}

\section{$2.1 \quad$ Introduction}

Let $H$ be a simple graph. A graphic sequence $\pi$ is said to be potentially $H$-graphic if it has a realization $G$ containing $H$ as its subgraph.

In 1991, Erdös, Jacobson and Lehel[3] considered the following problem about potentially $K_{k}$-graphic sequences: determine the smallest positive even number $\sigma(k, n)$ such that every $n$-term graphic sequence $\pi=\left(d_{1}, d_{2}, \cdots, d_{n}\right)$ without zero terms and with degree sum

$\sigma(\pi)=d_{1}+d_{2}+\cdots+d_{n}$ at least $\sigma(k, n)$ is potentially $K_{k}$-graphic. They gave a lower bound for $\sigma(k, n)$ by the example $\pi_{0}=\left((n-1)^{k-1},(k-1)^{n-k+1}\right)$, i.e., $\sigma(k, n) \geq(k-1)(2 n-k)+2$, and they further conjectured that the lower bound is the exact value of $\sigma(k, n)$. They also proved the conjecture is true for $k=2$ and $n \geq 6$, i.e., $\sigma(2, n)=2 n$ for $n \geq 6$. The conjecture is confirmed in [5], [21], [22] and [23] for any $k \geq 4$ and for $n$ sufficiently large. Li, Song and Luo[23] and Mubayi [26] also determined the values $\sigma(k, 2 k+2)$ independently. Recently, Li and Yin[20] determine the other values $\sigma(k, n)$.

In [5], Gould, Jacobson and Lehel generalized the problem: for a given simple graph $H$, determine the smallest positive even number $\sigma(H, n)$ such that every $n$-term graphic sequence $\pi=\left(d_{1}, d_{2}, \cdots, d_{n}\right)$ without zero terms and with degree sum $\sigma(\pi)=d_{1}+d_{2}+$ $\cdots+d_{n}$ at least $\sigma(H, n)$ is potentially $H$-graphic. They determined the values $\sigma\left(p K_{2}, n\right)$ and $\sigma\left(C_{4}, n\right)$ where $p K_{2}$ is the matching consisted of $p$ edges and $C_{4}$ is the cycle of length 4 .

In [16] and [17], C. H. Lai determined the values of $\sigma\left(C_{k}, n\right)$ for $k \geq 5$.

A graphic sequence is potentially ${ }_{3} C_{l}$-graphic if it has a realization containing a cycle of length $r$ for each $r, 3 \leq r \leq l$. 
In [18] and [19], $\mathrm{Li}$ and Luo determined the values $\sigma\left({ }_{3} C_{l}, n\right)$ for $l=4,5, \cdots, 9$ and $n \geq l$.

Motivated by the above problems, we consider the problem: characterize the potentially $H$-graphic sequences without zero terms.

In [28], Niu characterizes the potentially $\left(K_{k}-e\right)$-graphic sequences for $k=4,5$. Recently, Luo and Warner[25] characterized the potentially $K_{4}$-graphic sequences.

In this paper, we characterize the potentially $C_{k}$-graphic sequences for $k=3,4,5$. By these characterizations, the values $\sigma\left(K_{3}, n\right)$ and $\sigma\left(C_{k}, n\right)$ for $k=4,5$ are straightforward.

\subsection{Lemmas}

In order to prove our main results, we need the following results.

Let $\pi=\left(d_{1}, d_{2}, \cdots, d_{n}\right)$ be a nonincreasing positive integer sequence. Denote

$$
\pi^{\prime}=\left(d_{1}-1, d_{2}-1, \cdots, d_{d_{n}}-1, d_{d_{n}+1}, \cdots, d_{n-1}\right)
$$

From here on, $\pi^{\prime}$ will denote the residual sequence obtained by laying off $d_{n}$ from $\pi$ and all the graphic sequences have no zero terms.

The following theorem will be used repeatedly implicitly in the proof of our main theorem. We use $G \subseteq H$ to indicate that $G$ is a subgraph of $H$.

Theorem 2.2.1 (Gould, Jacobson and Lehel[5]) Let $\pi$ be a graphic sequence. If $H$ is a realization of $\pi$ with $G \subseteq H$, then there is a realization $H^{\prime}$ of $\pi$ with $G \subseteq H^{\prime}$ so that the vertices of $G$ have the largest degrees of $\pi$.

Lemma 2.2.2 (D. J. Kleitman and D. L. Wang [8] and Hakimi[6]) $\pi$ is graphic if and only if $\pi^{\prime}$ is graphic.

The following corollary is obvious.

Corollary 2.2.3 Let $H$ be a simple graph. If $\pi^{\prime}$ is potentially $H$-graphic, then $\pi$ is potentially H-graphic.

We will use Corollary 2.2.3 repeatedly in our proofs. 


\subsection{Potentially $C_{3}$-graphic Sequences}

The main result of this section is the following theorem.

Theorem 2.3.1 Let $\pi=\left(d_{1}, d_{2}, \cdots, d_{n}\right)$ be a graphic sequence with $n \geq 3$. Then $\pi$ is potentially $C_{3}$-graphic if and only if $d_{3} \geq 2$ except for 2 cases: $\pi=\left(2^{4}\right)$ and $\pi=\left(2^{5}\right)$.

Before we prove Theorem 2.3.1, we first apply the theorem to give a simple proof of the following theorem due to P. Erdös et al.

Theorem 2.3.2 (P. Erdös, M. S. Jacobson and J. Lehel [3])

$$
\sigma\left(K_{3}, n\right)=2 n \text { for } n \geq 6 .
$$

Proof. In [3], Erdös et al. gave the lower bound by the extremal example $D_{k}=((n-$ $\left.1)^{k-1},(k-1)^{n-k+1}\right)$, i.e., $\sigma\left(K_{k}, n\right) \geq(k-1)(2 n-k)+2$. Therefore, $\sigma\left(K_{3}, n\right) \geq 2 n$. We only need to show that $\sigma\left(K_{3}, n\right) \leq 2 n$. It is enough to show that any graphic sequence $\pi=\left(d_{1}, d_{2}, \cdots, d_{n}\right)$ with $\sigma(\pi) \geq 2 n$ is potentially $K_{3}$-graphic. Since $\sigma(\pi) \geq 2 n$, we must have that $d_{3} \geq 2$. By Theorem 2.3.1, $\pi$ is potentially $C_{3}$-graphic.

In order to prove Theorem 2.3.1, we need the following lemma.

Lemma 2.3.3 Let $\pi=\left(d_{1}, d_{2}, \cdots, d_{n}\right)$ be a graphic sequence with $d_{n} \geq 2$ and $n \geq 6$. Then $\pi$ is potentially $C_{3}$-graphic.

Proof. Since $\sigma(\pi) \geq 2 n$, every realization of $\pi$ must contain a cycle. Let $G$ be a realization of $\pi$ with minimal girth $g(G)$. Then $3 \leq g(G) \leq n$. We only need to show that $g(G)=3$.

By way of contradiction, we assume that $g(G) \geq 4$.

We consider the following 3 cases.

Case 1: $g(G) \geq 6$.

Let $v_{1} v_{2} v_{3} \cdots v_{g(G) v_{1}}$ be a cycle of length $g(G)$. Then $v_{1} v_{4}, v_{1} v_{5}, v_{2} v_{4}, v_{2} v_{5} \notin E(G)$. Therefore, $G^{\prime}=G-v_{1} v_{2}-v_{4} v_{5}+v_{1} v_{5}+v_{2} v_{4}$ is a realization of $\pi$ and $v_{2} v_{3} v_{4} v_{2}$ is a 3-cycle in $G^{\prime}$. This contradicts the minimality of $g(G)$.

Case 2: $g(G)=5$. Then $G$ has a cycle $C=v_{1} v_{2} v_{3} v_{4} v_{5} v_{1}$ of length 5 .

Subcase 2.1: $C$ is not a connected component of $G$.

By the assumption, there exits a vertex $u \neq v_{i}, i=1,2, \cdots, 5$ so that $u$ is adjacent to some vertex in $C$. 
Without loss of generality, we may assume that $u$ is adjacent to $v_{1}$. Since $g(G)=5$, $u v_{i} \notin E(G), i=2,3,4,5$. Also $v_{1} v_{4} \notin E(G)$, otherwise there is a cycle of length 3 or 4 in $G$. Therefore, $G^{\prime}=G-u v_{1}-v_{3} v_{4}+v_{1} v_{4}+u v_{3}$ is also a realization of $\pi$ and $G^{\prime}$ contains the cycle $v_{1} v_{5} v_{4} v_{1}$, which contradicts the minimality of $g(G)$.

Subcase 2.2: $C$ is a connected component of $G$.

By the assumption, for each $v_{i}, i=1,2,3,4,5$, is not adjacent to any vertices outside of $C$.

Since $n \geq 6$ and $d_{n} \geq 2$, there exists an edge $u v$ not on the cycle $C$. Therefore, $G^{\prime}=G-v_{1} v_{2}-v_{3} v_{4}-u v+v_{1} v_{4}+v_{2} u+v_{3} v$ is also a realization of $\pi$ with $g\left(G^{\prime}\right)=3$ since $v_{1} v_{4} v_{5} v_{1}$ is a 3 -cycle in $G^{\prime}$, which is a contradiction.

Case 3: $g(G)=4$. Let $C=v_{1} v_{2} v_{3} v_{4} v_{1}$ be a 4-cycle in $G$.

This case is similar to Case 2, we consider the following two subcases.

Subcase 3.1: $C$ is not a connected component of $G$.

By the assumption, there exists a vertex $u$ not in $C$ such that $u$ is adjacent to some $v_{i}$. Without loss of generality, we assume that $u$ is adjacent to $v_{1}$. Since $g(G)=4$, we must have that $v_{1} v_{3}, u v_{2} \notin E(G)$. Therefore, $G^{\prime}=G-v_{3} v_{2}-u v_{1}+v_{1} v_{3}+u v_{2}$ is also a realization of $\pi$ and the girth of $G^{\prime}$ is 3 since $v_{1} v_{3} v_{4} v_{1}$ is a 3 -cycle in $G^{\prime}$, which is a contradiction.

Subcase 3.2: $C$ is a connected component of $G$.

Since $n \geq 6$ and $d_{n} \geq 2$, there is an edge $u v$ not on the cycle $C$. Therefore, $G^{\prime}=$ $G-v_{1} v_{2}-v_{2} v_{3}-u v+v_{1} v_{3}+v_{2} u+v_{2} v$ is also a realization of $\pi$. Since $v_{1} v_{3} v_{4} v_{1}$ is a 3-cycle in $G^{\prime}$, the girth of $G^{\prime}$ is 3 , which is a contradiction.

Combining Case 1, 2 and $3, g(G)=3$.

Proof of Theorem 2.3.1 The necessary condition is obvious. Therefore, we only need to prove the sufficient condition. Let $\pi=\left(d_{1}, d_{2}, \cdots, d_{n}\right)$ be a graphic sequence satisfying the conditions of the theorem. It suffices to show that $\pi$ has a realization containing $C_{3}$ as its subgraph. We consider the following cases:

Case 1: $n=3$. This case is obvious.

Case 2: $n=4$. Since $\pi \neq\left(2^{4}\right), d_{3} \geq 2$ and $\sigma(\pi)$, we must have that $d_{1}=3$. Then $\pi$ must be one of the following sequences:

$$
(3,2,2,1),\left(3^{2}, 2^{2}\right),\left(3^{4}\right)
$$


It is easy to see that all of these are potentially $C_{3}$-graphic

Case 3. $n=5$. If $d_{1}=2$, since $\pi \neq\left(2^{5}\right), d_{3} \geq 2$ and $\sigma(\pi)$ is even, we must have $\pi=\left(2^{3}, 1^{2}\right)$. It is easy to see that it is potentially $C_{3}$-graphic. If $d_{1}=3$, then $\pi$ is one of the following:

$$
\left(3^{4}, 2\right),\left(3^{2}, 2^{3}\right),\left(3^{3}, 2,1\right),\left(3^{2}, 2,1^{2}\right),\left(3,2^{3}, 1\right) .
$$

It is easy to see that all of the above sequences are potentially $C_{3}$-graphic. If $d_{1}=4$, let $G$ be a realization of $\pi$ with vertices set $\left\{v_{1}, v_{2}, v_{3}, v_{4}, v_{5}\right\}$ where $d\left(v_{i}\right)=d_{i}, i=1,2, \cdots, 5$. Since $d\left(v_{1}\right)=4, v_{1}$ is adjacent to every other vertex. Since $d\left(v_{3}\right) \geq 2, v_{3}$ is adjacent to an vertex other than $v_{1}$, say $v_{i}$. Then $v_{1} v_{3} v_{i}$ forms a $C_{3}$.

Case $4: n \geq 6$.

We use induction to prove the sufficient condition.

First we prove the sufficient condition for $n=6$. Assume that $d_{3} \geq 2$.

If $d_{6} \geq 2$, by Lemma $2.3 .3, \pi$ is potentially $C_{3}$-graphic. Now we assume that $d_{6}=1$. Let $\pi^{\prime}=\left(d_{1}^{\prime}, d_{2}^{\prime}, d_{3}^{\prime}, d_{4}^{\prime}, d_{5}^{\prime}\right)$ be the residual sequence obtained by laying off $d_{6}$. If $d_{1} \geq 4$, then $d_{3}^{\prime} \geq \min \left\{d_{1}-1, d_{2}, d_{3}\right\} \geq 2$ and $d_{1}^{\prime} \geq 3$. By Case $3, \pi^{\prime}$ is potentially $C_{3}$-graphic. Therefore, $\pi$ is potentially $C_{3}$-graphic by Corollary 2.2.3. Thus, we may assume that $2 \leq d_{1} \leq 3$. If $d_{1}=2$, then $d_{1}=d_{2}=d_{3}=2$. Since $\sigma(\pi)$ is even, we must have that $d_{5}=1$ and $d_{4}=2$. Therefore, $\pi=\left(2^{4}, 1^{2}\right)$. It is easy to see that $\pi$ is potentially $C_{3}$-graphic. Now assume that $d_{1}=3$. If $d_{2}=3$, then $d_{1}^{\prime}=d_{2}=3$ and $d_{3}^{\prime} \geq \min \left\{d_{1}-1, d_{2}, d_{3}\right\} \geq 2$. Thus, by case $3, \pi^{\prime}$ is potentially $C_{3}$-graphic. Therefore, $\pi$ is potentially $C_{3}$-graphic by Corollary 2.2 .3 . Thus, we may further assume that $\pi=\left(3,2,2, d_{4}, d_{5}, 1\right)$. Since $\sigma(\pi)$ is even, $d_{4}+d_{5}$ must be even. Therefore, $\pi=\left(3,2^{4}, 1\right)$ or $\pi=\left(3,2^{2}, 1^{3}\right)$. It is easy to see that these both have realizations containing $C_{3}$. Therefore, $\pi$ is potentially $C_{3}$-graphic for $n=6$.

Now we suppose that the sufficient condition is true for $n-1 \geq 6$. We prove that it is also true for $n$. It suffices to prove that the graphic sequence $\pi=\left(d_{1}, d_{2}, \cdots, d_{n}\right)$ with $d_{3} \geq 2$ is potentially $C_{3}$-graphic by the definition.

If $d_{n} \geq 2$, by Lemma $2.3 .3, \pi$ is potentially $C_{3}$-graphic. Therefore, we may assume that $d_{n}=1$. Let $\pi^{\prime}=\left(d_{1}-1, d_{2}, d_{3}, \cdots, d_{n-1}\right)=\left(d_{1}^{\prime} d_{2}^{\prime}, \cdots, d_{n-1}^{\prime}\right)$ be the residual sequence obtained by lay ingoff $d_{n}=1$ where $d_{1}^{\prime} \geq d_{2}^{\prime} \geq \cdots \geq d_{n-1}^{\prime}$. If $d_{3}^{\prime} \leq 1$, then $d_{1}=d_{2}=d_{3}=2$ and $d_{4}=d_{5}=\cdots=d_{n}=1$. It follows that $\pi=\left(2^{3}, 1^{n-3}\right)$. Since $\sigma(\pi)$ is even, $n-3$ must be even. It follows that $\pi$ has a realization consisting of a $C_{3}$ and $(n-3) / 2$ disjoint edges. If $d_{3}^{\prime} \geq 2$, by induction hypothesis, $\pi^{\prime}$ is potentially $C_{3}$-graphic. Therefore, $\pi$ is potentially $C_{3}$-graphic by Corollary 2.2.3. 
This completes the proof.

\subsection{Potentially $C_{4}$-graphic Sequences}

The main result of this section is the following:

Theorem 2.4.1 Let $\pi=\left(d_{1}, d_{2}, \cdots, d_{n}\right)$ be a graphic sequence. Then $\pi$ is potentially $C_{4}$ graphic if and only if the following conditions hold:

(1) $d_{4} \geq 2$.

(2) $d_{1}=n-1$ implies $d_{2} \geq 3$.

(3) If $n=5,6$, then $\pi \neq\left(2^{n}\right)$.

Proof. First we assume that $\pi$ is potentially $C_{4}$-graphic. Then (1) and (3) are obvious. Assume that $d_{1}=n-1$. Let $G$ be a realization of $\pi$ that contains a $C_{4}$ as its subgraph and let $v_{1}$ be the vertex of $G$ with degree $d\left(v_{1}\right)=d_{1}$. Then $G-v_{1}$ contains a path with length at least 2 . Thus, $G-v_{1}$ has a vertex with degree at least 2 . Since $d\left(v_{1}\right)=n-1$, we must have $d_{2} \geq 3$.

Now we prove the sufficient condition.

We consider the following Cases:

Case 1. $n=4$.

If $d_{1}=2$, then $\pi=\left(2^{4}\right)$, which is potentially $C_{4}$-graphic. Now assume that $d_{1}=3$. Then $d_{2}=3$. Therefore, $\pi=\left(3^{2}, 2^{2}\right)$ or $\pi=\left(3^{4}\right)$. Obviously, both of these are potentially $C_{4}$-graphic..

Case 2. $n=5$.

If $d_{5} \geq 3$, then $\pi^{\prime}$ satisfies the assumption. Thus, $\pi^{\prime}$ is potentially $C_{4}$-graphic. Therefore, $\pi$ is potentially $C_{4}$-graphic by Corollary 2.2.3. Now we assume that $d_{5} \leq 2$. If $d_{5}=2$, consider $\pi^{\prime}$. If $d_{2} \geq 3$, then $\pi^{\prime}$ satisfies the assumption. Thus, $\pi^{\prime}$ is potentially $C_{4}$-graphic. If $d_{2}=2$, then $\pi=\left(d_{1}, 2^{4}\right)$. Since $\sigma(\pi)$ is even, we have that $d_{1}=2,4$, which is impossible. If $d_{5}=1$, then $d_{1} \geq 3$ since $d_{4} \geq 2$. Therefore, $\pi^{\prime}$ satisfies the assumption. Thus, $\pi$ is potentially $C_{4}$-graphic by Corollary 2.2.3.

Case 3. $n=6$.

If $d_{6} \geq 4$, then $\pi^{\prime}$ is potentially $C_{4}$-graphic. If $d_{6}=3$, then $\pi^{\prime}=\left(d_{1}-1, d_{2}-1, d_{3}-\right.$ $\left.1, d_{4}, d_{5}\right)$ satisfies the assumption. Thus, $\pi^{\prime}$ is potentially $C_{4}$-graphic. Therefore, $\pi$ is poten- 
tially $C_{4}$-graphic by Corollary 2.2.3. If $d_{6}=2$, then $\pi^{\prime}=\left(d_{1}-1, d_{2}-1, d_{3}, d_{4}, d_{5}\right)$ satisfies that $d_{4}^{\prime} \geq 2$ by condition (3). If $\pi^{\prime}=\left(2^{5}\right)$, then $\pi=\left(3^{2}, 2^{4}\right)$ and it is easy to see that $\pi$ is potentially $C_{4}$-graphic. If $d_{1}^{\prime}=4$ and $d_{2}^{\prime} \leq 2$, then $d_{2}^{\prime}=2$. Therefore, $\pi^{\prime}=\left(4,2^{k}, 1^{5-k-1}\right)$ where $k \geq 3$. Since $\sigma\left(\pi^{\prime}\right)$ is even, $k=4$. Thus, $\pi^{\prime}=\left(4,2^{4}\right)$. Therefore, $\pi=\left(5,3,2^{4}\right)$ and it is easy to see that $\pi$ is potentially $C_{4}$-graphic. If $d_{6}=1$, then $\pi^{\prime}=\left(d_{1}-1, d_{2}, d_{3}, d_{4}, d_{5}\right)$. If $d_{4}^{\prime}=1$, then $\pi=\left(2^{4}, 1^{2}\right)$. Obviously, $\pi$ is potentially $C_{4}$-graphic. Now we assume that $d_{4}^{\prime} \geq 2$. If $\pi^{\prime} \neq\left(2^{5}\right)$ or $\left(4,2^{4}\right)$, then $\pi^{\prime}$ is potentially $C_{4}$-graphic. Therefore, $\pi$ is potentially $C_{4}$-graphic by Corollary 2.2.3. If $\pi^{\prime}=\left(2^{5}\right)$, then $\pi=\left(3,2^{4}, 1\right)$. Clearly $\pi$ is potentially $C_{4}$-graphic. If $\pi^{\prime}=\left(4,2^{4}\right)$, then $\pi=\left(5,2^{4}, 1\right)$, which is impossible by $(2)$.

Case $4: n \geq 7$.

We use induction to prove this case.

First we show the case for $n=7$. If $d_{7} \geq 3$, by Case 3 , we are done. We assume that $d_{7} \leq 2$. If $d_{7}=2$, then $d_{4}^{\prime} \geq 2$. If $d_{7}=1$, then $d_{4}^{\prime} \geq 2$ otherwise $\pi=\left(2^{4}, 1^{3}\right)$. Therefore, $d_{4}^{\prime} \geq 2$ if $d_{7} \leq 2$. If $d_{1}^{\prime}=5$ and $d_{2}^{\prime}=2$, then $\pi^{\prime}=\left(5,2^{4}, 1\right)$. Therefore, $d_{1}=6$ and $d_{2}=2$, which contradicts the assumption. Thus, if $\pi^{\prime} \neq\left(2^{6}\right)$, then $\pi^{\prime}$ is potentially $C_{4}$-graphic. Therefore, $\pi$ is potentially $C_{4}$-graphic by Corollary 2.2.3. If $\pi^{\prime}=\left(2^{6}\right)$, then $\pi=\left(3^{2}, 2^{5}\right)$ or $\pi=\left(3,2^{5}, 1\right)$. Clearly they are potentially $C_{4}$-graphic. It follows that the sufficient condition is true for $n=7$

Now we assume that the sufficient condition is true for $n-1 \geq 7$. We prove that it is true for $n$. Let $\pi=\left(d_{1}, \cdots, d_{n}\right)$ be a graphic sequence with $n$ terms that satisfies the conditions (1) and (2). We only need to show that $\pi$ is potentially $C_{4}$-graphic. If $d_{n} \geq 3$, then $\pi^{\prime}$ satisfies the assumption. By induction hypothesis, $\pi^{\prime}$ is potentially $C_{4}$-graphic. Therefore, $\pi$ is potentially $C_{4}$-graphic by Corollary 2.2.3. If $d_{n}=2$, then $\pi^{\prime}=\left(d_{1}-1, d_{2}-1, d_{3}, \cdots, d_{n-1}\right)$. Then $d_{4}^{\prime} \geq 2$. If $d_{1}^{\prime}=n-2$, then either $d_{1}=n-1$ or $d_{1}=d_{2}=d_{3}=n-2$. In the later case, $\pi^{\prime}$ satisfies the assumption. By the induction hypothesis, $\pi^{\prime}$ is potentially $C_{4}$-graphic. Therefore, $\pi$ is potentially $C_{4}$-graphic by Corollary 2.2.3. If $d_{1}=n-1$, then either $\pi^{\prime}$ satisfies the assumption or $\pi=\left(n-1,3,2^{n-2}\right)$. It is easy to see that $\left(n-1,3,2^{n-2}\right)$ is potentially $C_{4}$-graphic. Now we assume that $d_{n}=1$. If $d_{4}^{\prime}=1$, then $\pi=\left(2^{4}, 1^{n-4}\right)$. Then $n$ is even and it is easy to see $\pi$ is potentially $C_{4}$-graphic. Now assume that $d_{4}^{\prime} \geq 2$. If $d_{1}^{\prime}=n-2$ and $d_{2}^{\prime}=2$, then $d_{1}=n-1$ and $d_{2}=2$, which contradicts the assumption. Therefore, $\pi^{\prime}$ is potentially $C_{4}$-graphic. Hence $\pi$ is potentially $C_{4}$-graphic by Corollary 2.2.3.

By Theorem 2.4.1, we give a simple proof of the following theorem due to R.J. Gould et al.: 
Theorem 2.4.2 (R. J. Gould, M. S. Jacobson, J. Lehel[5])

For $n \geq 4$,

$$
\sigma\left(C_{4}, n\right)= \begin{cases}3 n-1, & \text { if } n \text { is odd } \\ 3 n-2, & \text { if } n \text { is even }\end{cases}
$$

Proof. In [5], by taking the extremal examples $\pi=\left((2 k)^{1}, 2^{2 k}\right)$ when $n=2 k+1$ and $\pi=\left((2 k+1)^{1}, 2^{2 k}, 1\right)$ when $n=2 k, \mathrm{R}$. J. Gould et al. presented a lower bound for $\sigma\left(C_{4}, n\right)$, i.e.,

$$
\sigma\left(C_{4}, n\right) \geq \begin{cases}3 n-1, & \text { if } n \text { is odd } \\ 3 n-2, & \text { if } n \text { is even }\end{cases}
$$

We show that

$$
\sigma\left(C_{4}, n\right) \leq \begin{cases}3 n-1, & \text { if } n \text { is odd } \\ 3 n-2, & \text { if } n \text { is even }\end{cases}
$$

Let $\pi$ be a graphic sequence with $\sigma(\pi) \geq 3 n-2$. It suffices to show that $\pi$ is potentially $C_{4}$-graphic. If $d_{4}=1$, then $\sigma(\pi)=d_{1}+d_{2}+d_{3}+(n-3)$ and $d_{1}+d_{2}+d_{3} \leq 6+(n-3)=n+3$. Therefore, $\sigma(\pi) \leq 2 n \leq 3 n-2$. Thus, $d_{4} \geq 2$. Clearly, if $n=5,6, \pi \neq\left(2^{n}\right)$. Assume that $d_{1}=n-1$. If $d_{2} \leq 2$, then $\sigma(\pi) \leq n-1+2(n-1)=3 n-3 \leq 3 n-2$. Therefore, $\pi$ satisfies the conditions (1)-(3) in Theorem 2.4.1, and thus, $\pi$ is potentially $C_{4}$-graphic.

\subsection{Potentially $C_{5}$-graphic Sequences}

The main result of this section is the following:

Theorem 2.5.1 Graphic sequence $\pi=\left(d_{1}, d_{2}, \cdots, d_{n}\right)$ is potentially $C_{5}$-graphic if and only if $\pi$ satisfies the following conditions:

(1) $d_{5} \geq 2$ and $\pi \neq\left(2^{n}\right)$ for $n=6,7$.

(2) For $i=1,2, d_{1}=n-i$ implies $d_{4-i} \geq 3$.

(3) If $\pi=\left(d_{1}, d_{2}, 2^{k}, 1^{n-k-2}\right)$, then $d_{1}+d_{2} \leq n+k-2$.

Proof. We assume that $\pi$ is potentially $C_{5}$-graphic. It is obvious that (1) holds. Assume $\pi=\left(d_{1}, d_{2}, 2^{k}, 1^{n-k-2}\right)$ with $d_{1}+d_{2} \geq n+k$. Since $\pi$ is graphic, $d_{1}+d_{2} \leq 2 k+n-k-2+2=$ $n+k$. Therefore, $d_{1}+d_{2}=n+k$. Hence $\pi$ has a unique realization. It is easy to see that the realization is of girth 4 . Hence (3) holds. Let $G$ be a realization of $\pi$ that contains a 
cycle $C_{5}$ and let $v_{1}$ be the vertex of $G$ with degree $d\left(v_{1}\right)=d_{1}$. Then $G-v_{1}$ contains a path with length at least 3 . Thus, $G-v_{1}$ contains at least two vertices with degree at least 2 . Therefore, $d_{1}=n-i, i=1,2$ implies $d_{4-i} \geq 3$. Thus, (2) holds.

Now we prove the sufficient condition. It is enough to show the following three lemmas.

Lemma 2.5.2 Graphic sequence $\pi=\left(d_{1}, d_{2}, \cdots, d_{5}\right)$ is potentially $C_{5}$-graphic if $\pi$ satisfies the following conditions:

(1) $d_{5} \geq 2$;

(2) If $d_{1}=4$, then $d_{3} \geq 3$.

Proof. If $d_{5} \geq 3$, then, by Theorem $2.4 .1, \pi^{\prime}$ is potentially $C_{4}$-graphic. Since $d_{5} \geq 3, \pi$ is potentially $C_{5}$-graphic. Now assume that $d_{5}=2$. If $d_{1}=2$, then $\pi=\left(2^{5}\right)$. Obviously this is potentially $C_{5}$-graphic. If $d_{1}=3$, then $\pi=\left(3^{2}, 2^{3}\right)$ or $\pi=\left(3^{4}, 2\right)$. It is easy to see that these are potentially $C_{5}$-graphic. Now assume that $d_{1}=4$, then $d_{3} \geq 3$ by (2). Since $d_{5}=2$, we have $d_{3}=3$. Therefore, $\pi$ is $\left(4^{2}, 3^{3}, 2\right)$ or $\left(4,3^{2}, 2^{2}\right)$. It is easy to see that these are potentially $C_{5}$-graphic.

Lemma 2.5.3 For $n=6,7, \pi=\left(d_{1}, d_{2}, \cdots, d_{n}\right)$ is potentially $C_{5}$-graphic if $\pi$ satisfies the following conditions:

(1) $d_{5} \geq 2$ and $\pi \neq\left(2^{n}\right)$.

(2) For $i=1,2, d_{1}=n-i$ implies $d_{4-i} \geq 3$.

(3) $\pi=\left(d_{1}, d_{2}, 2^{k}, 1^{n-k-2}\right)$ implies $d_{1}+d_{2} \leq n+k-2$.

Proof. We first prove the case for $n=6$. Notice that if $d_{6} \geq 3$, then $\pi^{\prime}$ satisfies the conditions (1) and (2) in Lemma 2.5.2. Hence by Lemma $2.5 .2, \pi^{\prime}$ is potentially $C_{5}$-graphic. Therefore, $\pi$ is potentially $C_{5}$-graphic by Corollary 2.2 .3 . Thus, we may assume that $d_{6} \leq 2$.

We consider the following two cases:

Case 1: $d_{6}=1$. In this case, $d_{1} \geq 3$ otherwise $\sigma(\pi)$ is odd. If $d_{3} \geq 3$, then by Lemma 2.5.2, $\pi^{\prime}$ is potentially $C_{5}$-graphic. Now we assume that $d_{3}=2$. Then $\pi=\left(d_{1}, d_{2}, 2^{3}, 1\right)$. Then by conditions (3) and (2), $d_{1} \leq n-2=4$ and $d_{1}+d_{2} \leq n+k-2=6+3-2=7$. Since $d_{1} \geq 3$, $5 \leq d_{1}+d_{2} \leq 7$. Since $\sigma(\pi)$ is even, $d_{1}+d_{2}=5$ or 7 . Thus, $\pi=\left(3,2^{4}, 1\right)$ or $\left(4,3,2^{3}, 1\right)$ and it is easy to see that they are potentially $C_{5}$-graphic. 
Case 2: $d_{6}=2$. By (1), $d_{1} \geq 3$. If $d_{5} \geq 3$ or $d_{3} \geq 4$, then by Lemma $2.5 .2, \pi^{\prime}$ is potentially $C_{5}$-graphic. Therefore, $\pi$ is potentially $C_{5}$-graphic by Corollary 2.2.3. Thus, we may assume that $d_{5}=2$ and $d_{3} \leq 3$. If $d_{3}=2$, then $\pi=\left(d_{1}, d_{2}, 2^{4}\right)$. Hence by (2) and (3), we have that $d_{1}+d_{2} \leq n+k-2=6+4-2=8$ and $d_{1} \leq 4$. Thus, $\pi=\left(4^{2}, 2^{4}\right)$ or $\left(3^{2}, 2^{4}\right)$. It is easy to see both of these are potentially $C_{5}$-graphic. If $d_{3}=3$, then we may further assume that $d_{2}=3$ otherwise by Lemma $2.5 .2, \pi^{\prime}$ is potentially $C_{5}$-graphic. Then $\pi$ is one of the following sequences:

$$
\left(5,3^{3}, 2^{2}\right),\left(4,3^{2}, 2^{2}\right),\left(4,3^{4}, 2\right),\left(3^{4}, 2^{2}\right)
$$

It is easy to see that these are potentially $C_{5}$-graphic.

Now we prove the case for $n=7$.

Similar to the $n=6$ case, we may assume that $d_{7} \leq 2$. If $d_{3} \geq 4$ or $d_{5} \geq 3$, then by the case for $n=6, \pi^{\prime}$ is potentially $C_{5}$-graphic. Therefore, we may further assume that $d_{3} \leq 3$ and $d_{5}=2$. We consider the following two cases:

Case 1. $d_{7}=1$. We may assume that $d_{4}=2$, otherwise $\pi^{\prime}$ is potentially $C_{5}$-graphic. If $d_{3}=3$, then we may assume that $d_{1}=d_{2}=d_{3}=3$, otherwise $\pi^{\prime}$ is potentially $C_{5^{-}}$ graphic. Therefore, $\pi=\left(3^{3}, 2^{3}, 1\right)$ and it is potentially $C_{5}$-graphic. If $d_{3}=2$, then by $(2)$ and (3), $d_{1} \leq 5$ and $d_{1}+d_{2} \leq n+k-2 \leq 7+4-2=9$. Then $\pi$ is one of the following sequences:

$$
\begin{gathered}
\left(5,4,2^{4}, 1\right),\left(4,3,2^{4}, 1\right),\left(3,2^{5}, 1\right),\left(5,3,2^{3}, 1^{2}\right), \\
\left(4^{2}, 2^{3}, 1^{2}\right),\left(4,2^{4}, 1^{2}\right),\left(3^{2}, 2^{3}, 1^{2}\right),\left(2^{5}, 1^{2}\right) .
\end{gathered}
$$

It is easy to see that these are all potentially $C_{5}$-graphic.

Case 2: $d_{7}=2$. In this case $d_{1} \geq 3$. If $d_{3}=3$, then similar to Case 1 , we may assume that $d_{2}=d_{3}=3$. Therefore, $\pi$ is one of the following sequences: $\left(3^{4}, 2^{3}\right),\left(5,3^{3}, 2^{3}\right),\left(6,3^{2}, 2^{4}\right),\left(4,3^{2}, 2^{4}\right)$ and it is easy to see that these are potentially $C_{5}$-graphic. If $d_{3}=2$, then by (2) and (3), $d_{1} \leq 5$ and $d_{1}+d_{2} \leq n+k-2=7+5-2=10$. Then $\pi$ is one of the following sequences: $\left(5^{2}, 2^{5}\right),\left(5,3,2^{5}\right),\left(4^{2}, 2^{5}\right),\left(4,2^{6}\right),\left(3^{2}, 2^{5}\right)$ and it is easy to see that these are potentially $C_{5^{-}}$ graphic.

Lemma 2.5.4 For $n \geq 8, \pi=\left(d_{1}, d_{2}, \cdots, d_{n}\right)$ is potentially $C_{5}$-graphic if $\pi$ satisfies the following conditions: 
(1) $d_{5} \geq 2$

(2) For $i=1,2, d_{1}=n-i$ implies $d_{4-i} \geq 3$.

(3) $\pi=\left(d_{1}, d_{2}, 2^{k}, 1^{n-k-2}\right)$ implies $d_{1}+d_{2} \leq n+k-2$.

Proof. We are going to prove this lemma by induction on $n$.

We first prove the lamma for $n=8$. We may assume that $d_{8} \leq 2$, otherwise, by Lemma 2.5.3, $\pi^{\prime}$ is potentially $C_{5}$-graphic. Similar to the proof of Lemma 2.5.3, we may further assume that $d_{3} \leq 3$ and $d_{5}=2$. We consider the following two cases:

Case $1 . d_{8}=1$. If $d_{3}=3$, then we may assume that $d_{1}=d_{2}=d_{3}=3$ and $d_{4}=2$ otherwise $\pi^{\prime}$ is potentially $C_{5}$-graphic by Lemma 2.5.3. Therefore, $\pi=\left(3^{3}, 2^{4}, 1\right)$ or $\left(3^{3}, 2^{2}, 1^{3}\right)$ and it is easy to see that these are potentially $C_{5}$-graphic. Now we assume that $d_{3}=2$. Then $\pi=\left(d_{1}, d_{2}, 2^{k}, 1^{8-k-2}\right)$. Then by (2) and (3), $d_{1} \leq 6$ and $d_{1}+d_{2} \leq n+k-2$. If $d_{1}=2$, then $\pi=\left(2^{6}, 1^{2}\right)$. It is easy to see that this is potentially $C_{5}$-graphic. Thus, we may further assume that $d_{1} \geq 3$. Then $\pi^{\prime}=\left(d_{1}-1, d_{2}, 2^{k}, 1^{n-1-k-2}\right)$ and $d_{1}-1+d_{2} \leq n+k-2-1=$ $n-1+k-2$. If $\pi^{\prime}=\left(2^{7}\right)$, then $\pi=\left(3,2^{6}, 1\right)$ and it is easy to see that this is potentially $C_{5}$-graphic. Now we assume that $\pi^{\prime} \neq\left(2^{7}\right)$. Since $d_{1} \leq 6, \pi^{\prime}$ satisfies the condition (2) in Lemma 2.5.3. If $\pi^{\prime} \neq\left(2^{7}\right)$, then it satisfies the conditions (1), (2) and (3) in Lemma 2.5.3. By Lemma $2.5 .3, \pi^{\prime}$ is potentially $C_{5}$-graphic. Therefore, $\pi$ is potentially $C_{k}$-graphic by Corollary 2.2.3.

Case 2. $d_{8}=2$. If $d_{3}=2$, then $\pi=\left(d_{1}, d_{2}, 2^{6}\right)$. By (2) and (3), $d_{1} \leq 6$ and $d_{1}+d_{2} \leq$ $n+k-2=8+6-2=12$. If $d_{2}=2$, then $\pi=\left(4,2^{7}\right)$ or $\left(2^{8}\right)$. It is easy to see that these are potentially $C_{5}$-graphic. Thus, we may further assume that $d_{2} \geq 3$. Then $\pi^{\prime}=\left(d_{1}-1, d_{2}-1,2^{5}\right)$. If $\pi^{\prime}=\left(2^{7}\right)$, then $\pi=\left(3^{2}, 2^{6}\right)$. It is easy to see that this is potentially $C_{5}$-graphic. Therefore, we may assume that $\pi^{\prime} \neq\left(2^{7}\right)$. Thus, $\pi^{\prime}$ satisfies the condition (1) in Lemma 2.5.3. Since $d_{1} \leq 6, \pi^{\prime}$ satisfies the condition (2) in Lemma 2.5.3. Since $d_{1}^{\prime}+d_{2}^{\prime}=d_{1}-1+d_{2}-1 \leq 12-2=10=7+5-2, \pi^{\prime}$ satisfies the condition (3) in Lemma 2.5.3. Therefore, by Lemma $2.5 .3, \pi^{\prime}$ is potentially $C_{5}$-graphic. Therefore, $\pi$ is potentially $C_{k}$-graphic by Corollary2.2.3. If $d_{3}=3$, then we may assume that $d_{2}=3$, otherwise $\pi^{\prime}$ is potentially $C_{5}$-graphic. Thus, $\pi$ is one of the following sequences:

$$
\left(3^{4}, 2^{4}\right),\left(5,3^{3}, 2^{4}\right),\left(7,3^{3}, 2^{4}\right),\left(6,3^{2}, 2^{5}\right),\left(4,3^{2}, 2^{5}\right) .
$$

It is easy to see that these are all potentially $C_{5}$-graphic.

Now we assume that the lemma is true for $n-1 \geq 8$. We show it is true for $n$. Let $\pi=\left(d_{1}, d_{2}, \cdots, d_{n}\right)$ be a graphic sequence satisfying the conditions (1)-(3). We need only 
show that $\pi$ is potentially $C_{5}$-graphic. If $d_{n} \geq 3$, then, by induction hypothesis, $\pi^{\prime}$ is potentially $C_{5}$-graphic and therefore, $\pi$ is potentially $C_{5}$-graphic by Corollary 2.2.3. Thus, we may assume that $d_{n} \leq 2$. Similarly, we may further assume that $d_{d_{n}+3}=2$ and $d_{3} \leq 3$. We consider the following two cases:

Case 1. $d_{n}=1$. In this case $d_{4}=2$. If $d_{1}=2$, then $\pi=\left(2^{k}, 1^{n-k}\right)$. Since $k \geq 5$ and $n \geq 9, \pi$ is potentially $C_{5}$-graphic. Thus, we further assume that $d_{1} \geq 3$. If $d_{3}=3$, then we may assume that $d_{1}=d_{2}=d_{3}=3$. Then $\pi^{\prime}=\left(3^{2}, 2^{k+1}, 1^{n-k-4}\right)$ satisfies (1),(2) and (3) and therefore, $\pi^{\prime}$ is potentially $C_{5}$-graphic and hence $\pi$ is potentially $C_{5}$-graphic. If $d_{3}=2$, then $\pi=\left(d_{1}, d_{2}, 2^{k}, 1^{n-k-2}\right)$. Therefore, $\pi^{\prime}=\left(d_{1}-1, d_{2}, 2^{k}, 1^{n-k-2}\right)$. By (3), $d_{1}+d_{2} \leq n+k-2$. Thus, $d_{1}-1+d_{2} \leq n-1+k-2$. Obviously, $\pi^{\prime}$ satisfies (1)-(3). So $\pi^{\prime}$ is potentially $C_{5}$-graphic and hence $\pi$ is potentially $C_{5}$-graphic.

Case 2. $\quad d_{n}=2$. In this case $d_{5}=2$. Therfore $\pi=\left(d_{1}, d_{2}, d_{3}, d_{4}, 2^{n-4}\right)$. If $d_{3}=2$, then $\pi=\left(d_{1}, d_{2}, 2^{n-2}\right)$. By (2) and (3), $d_{1}+d_{2} \leq n+n-2-2=2(n-2)$ and $d_{1} \leq n-2$. If $d_{2} \geq 4$ or $d_{1} \leq n-3$ and $d_{2} \geq 3$, then $\pi^{\prime}$ satisfies (1)-(3) and therefore, by induction hypothesis it is potentially $C_{5}$-graphic and hence $\pi$ is potentially $C_{5}$-graphic. If $\pi=\left(n-2,3,2^{n-2}\right)$, it is easy to see that $\pi$ is potentially $C_{5}$-graphic. Now assume that $d_{2}=2$. If $d_{1}=2$, then $\pi$ is potentially $C_{5}$-graphic since $n \geq 9$. If $d_{1} \geq 3$, then by (3)and (2), $d_{1}-1+2 \leq n-3+2-2=n-3 \leq n-1+n-3-2=2 n-5$ and $d_{1}-1 \leq n-3-1=(n-1)-3$. It follows that $\pi^{\prime}$ is potentially $C_{5}$-graphic and hence $\pi$ is potentially $C_{5}$-graphic. Now assume that $d_{3}=3$. If $d_{4}=3$, then we may assume that $d_{1}=3$ otherwise $\pi^{\prime}$ is potentially $C_{5}$ graphic. Thus, $\pi=\left(3^{4}, 2^{n-4}\right)$ and is potentially $C_{5}$-graphic since $n \geq 9$. Now we assume that $d_{4}=2$. Then $\pi=\left(d_{1}, d_{2}, 3,2^{n-3}\right)$. Similarly we may assume that $d_{2}=3$. If $d_{1}=n-1$, then $\pi$ is potentially $C_{5}$-graphic. If $d_{1} \leq n-2$, then $d_{1}^{\prime}=d_{1}-1 \leq(n-1)-2$ and $d_{1}^{\prime}+d_{2}^{\prime}=d_{1}-1+3 \leq n-2-1+3=n \leq n-1+n-3-2=2 n-6$. Thus, $\pi^{\prime}$ is potentially $C_{5}$-graphic and therefore, $\pi$ is potentially $C_{5}$-graphic.

Therefore, $\pi$ is potentially $C_{5}$-graphic.

Now we use Theorem 2.5.1 to give a simple proof of a theorem due to C.H. Lai.

Theorem 2.5.5 (C.H. Lai [16]) $\sigma\left(C_{5}, n\right)=4 n-4$ for $n \geq 5$.

Proof. Take $\pi=\left((n-1)^{2}, 2^{(n-2)}\right)$. Then $\pi$ has unique realization and this realization doesn't contain a cycle of length 5 . Therefore, $\sigma\left(C_{5}, n\right) \geq \sigma(\pi)+2=4 n-4$. Now we will show that $\sigma\left(C_{5}, n\right) \leq 4 n-4$. Let $\pi$ be a graphic sequence with $\mathrm{n}$ terms and with $\sigma(\pi) \geq 4 n-4$. It suffices to show that $\pi$ is potentially $C_{5}$-graphic. If $d_{5}=1$, then 
$d_{1}+d_{2}+d_{3}+d_{4} \leq 4 \times 3+n-4=n+8$. Therefore, $\sigma(\pi) \leq n+8+(n-4)=2 n+4 \leq 4 n-4$, since $n \geq 5$, which is a contradiction. Thus, $d_{5} \geq 2$. If $d_{3}=2$, then $4 n-4 \leq \sigma(\pi) \leq$ $d_{1}+d_{2}+d_{3}(n-2) \leq 2(n-1)+2(n-2)=4 n-6$. The contradiction shows that $d_{3} \geq 3$. Therefore, $\pi$ satisfies the conditions in Theorem 2.5.1. By Theorem 2.5.1, $\pi$ is potentially $C_{4}$-graphic. Thus, $\sigma\left(C_{5}, n\right)=4 n-4$ for $n \geq 5$.

\subsection{Conclusion}

It would be nice to characterize potentially $C_{k}$-graphic sequences for general $k$. This question seems very difficult. The special case, characterizing potentially Hamiltonian graphic sequences, has been open for several decades. We believe that new techniques are needed to slove this problem. 


\section{Bibliography}

[1] J. Edmonds, Existence of $k$-edge connected ordinary graphs with prescribed degree, $J$. Res. Nat. Bur. Stand. Ser. B, 68(1964), 73-74.

[2] P. Erdos, T. Gallai, Graphs with given degrees of vertices (Hungarian), Math. Lapok, 11(1960), 264-274.

[3] P. Erdös, M. S. Jacobson, J. Lehel, Graphs realizing the degree sequences and their respective clique numbers. In: Y. Alavi et al ed, Graph Theory, Combinatorics \& Applications Vol.1, New York, John Wiley \& Sons Inc, 1991, 439-449.

[4] D.A. Gale, A theorem on flows in networks, Pacific J. Math., 7(1957), 1073-1083.

[5] R. J. Gould, M. S. Jacobson, J. Lehel, Potentially G-graphic degree sequences, to appear.

[6] S. L. Hakimi, On realizability of a set of integers and the degrees of the vertices of a linear graph, I, II, SIAM J. Appl. Math, 10(1962), 496-506; 11(1963), 135-147.

[7] V. Havel, A remark on the existence of finite graphs (Hungarian), Casopis P. Math., 80(1995), 477-480.

[8] D. J. Kleitman, D. L. Wang, Algorithm for constructing graphs and digraphs with given valences and factors, Discrete Math, 6(1)(1973), 79-88.

[9] R. H. Johnson, The diameter and radius of simple graphs, J. Combin. Theory Ser. B., 17(1974), 188-198.

[10] R. H. Johnson, Simple separable graphs, Pacific J. Math., 56(1975), 143-158.

[11] R. H. Johnson, Simple directed trees, Discrete Math., 14(1976), 257-264.

[12] D. J. Kleitman and S. Y. R. Li, A note on unigraphic sequences, Stud. Appl. Math., $54(4)(1975), 283-287$. 
[13] M. Koren, Extreme degree sequences of simple graphs, J. Combin. Theory Ser. B., 159(1973), 213-224.

[14] M. Koren, Pairs of sequences with a unique realization by bipartite graphs, Ibid., 21(1976), 224-234.

[15] M. Koren, Sequences with unique realization by simple graphs, Ibid, 21(1976), 235-244.

[16] Chun-Hui Lai, Potentially $C_{k}$-graphic sequences, Journal of Zhang Zhou Teachers College (in Chinese), 4 (1997), 27-31.

[17] Chun-Hui Lai, The smallest degree sum that yields potentially $C_{k}$-graphic sequences, submitted.

[18] Jiongsheng Li and Rong Luo, Potentially ${ }_{3} C_{l}$-graphic sequences, J. Univ. Sci. Technol. China, 29(1)(1999), 1-8.

[19] Jiongsheng Li, Rong Luo and Yunkai Liu, An extremal problem on potentially ${ }_{3} C_{l^{-}}$ graphic sequences, J. Math. Study 31(4)(1998), 362-369.

[20] Jiongsheng Li and Jiahua Yin, The threshold for the Erdös, Jacobson and Lehel conjecture being true, preprint.

[21] Jiong-Sheng Li, Zi-Xia Song, An extremal problem on the potentially $P_{k}$-graphic degree sequences, Discrete Math., 212(3)(2000), 223-231.

[22] Jiong-Sheng Li,Zi-Xia Song, The smallest degree sum that yields potentially $P_{k}$-graphic sequences, J. Graph Theory, 29(1998), 63-72.

[23] Jiong-Sheng Li, Zi-Xia Song and Rong Luo, The Erdös-Jacobson-Lehel conjecture on potentially $P_{k}$-graphic sequences is true, Science in China, 41(5)(1998), 510-520.

[24] S. Y. R. Li, Graphic sequences with unique realization, J. Combin. Theory Ser. B., 42(1)(1975), 42-68.

[25] Rong Luo and Morgan Warner, On potentially $K_{k}$-graphic sequences, preprint.

[26] D. Mubayi, Graphic sequences that have a realization with large clique number, $J$. Graph Theory, 34(2000), 20-29.

[27] C. St. J. A. Nash-Williams, Valency sequences which force graphs to have Hamiltonian circuits, Interim report, University of Waterloo, preprint (1970). 
[28] Jianbin Niu, On Potentially $\left(K_{4}-e\right)$-graphic sequences, preprint.

[29] S. B. Rao and A. R. Rao, Existence of 3-connected graphs with prescribed degrees, Pacific J. Math., 33(1970), 203-207.

[30] J. K. Senion, Partitions and their representating graphs, Amer. J. Math., 73(1951), 663-689.

[31] G. Sierksma and Hoogeveen, Seven criteria for integer sequences being graphic, J. Graph Theory, 15(1991), 223-231.

[32] D. L. Wang and D. J. Kleitman, On the existence of $n$-connected graphs with prescribed degree ( $n \geq 2)$, Networks, 3(1973), 225-239. 